\title{
Characterization of Dutch Staphylococcus aureus from bovine mastitis using a Multiple Locus Variable Number Tandem Repeat Analysis
}

\author{
Risma Ikawaty ${ }^{a}$, E.C. Brouwer ${ }^{a}$, M.D. Jansen ${ }^{a}$, E. van Duijkeren ${ }^{\text {b }}$, D. Mevius ${ }^{\text {b,c }}$, \\ J. Verhoef ${ }^{a}$, A.C. Fluit ${ }^{\mathrm{a}, *}$ \\ ${ }^{a}$ Department of Medical Microbiology, University Medical Centre Utrecht, Utrecht, The Netherlands \\ ${ }^{\mathrm{b}}$ Faculty of Veterinary Medicine, Utrecht University, Utrecht, The Netherlands \\ ${ }^{\mathrm{c}}$ Central Veterinary Institute, Lelystad, The Netherlands
}

\section{A R T I C L E I N F O}

\section{Article history:}

Received 15 July 2008

Received in revised form 24 October 2008

Accepted 29 October 2008

\section{Keywords:}

Staphylococcus aureus

Bovine

Mastitis

MLVA

MLST

PFGE

Spa-typing

\begin{abstract}
A B S T R A C T
Current typing methods for Staphylococcus aureus have important drawbacks. We evaluated a Multiple Locus Variable Number Tandem Repeat Analysis (MLVA) scheme with 6 loci which lacks most drawbacks on 85 bovine mastitis isolates from The Netherlands. For each locus the number of repeat units (RU) was calculated. Each combination of repeat units was assigned a MLVA-type (MT). We compared the MLVA typing result with Multi Locus Sequence Typing (MLST), spa-typing and Pulsed-Field Gel Electrophoresis (PFGE). MLVA typing resulted in 18 MTs, although 3 loci could not always be amplified. Spa-typing distinguished 10 spa-types including 3 dominant and 2 new types. PFGE showed 5 dominant profiles with 15 related profiles and 6 unique profiles. MLST showed 4 dominant STs. Some types appeared to be bovine specific. The Simpson's Indices of diversity for PFGE, MLST, spa-typing and MLVA were 0.887, 0.831, 0.69 and 0.781 , respectively, indicating that discriminatory power of MLVA was between MLST and spa-typing, whereas PFGE displayed the highest discriminatory power. However, MLVA is fast and cheap when compared to the other methods. The Adjusted Rand index and Wallace's coefficient indicated that MLVA was highly predictive for spa-type, but not vice versa.

Analysis of the region neighboring SIRU05 showed a difference in the genetic element bordering the repeats of SIRU05 that explained the negative SIRU05 PCRs. PFGE, MLST, and MLVA are adequate typing methods for bovine-associated $S$. aureus.
\end{abstract}

(c) 2008 Elsevier B.V. All rights reserved.

\section{Introduction}

Staphylococcus aureus is a major agent of contagious mastitis in diary cattle. The sources of bovine mastitis cases mostly are from bovine origin, but $S$. aureus originating from the farmer are another important source (Zadoks et al., 2002). In 2005 it was reported that each year

\footnotetext{
* Corresponding author at: Department of Medical Microbiology, University Medical Centre Utrecht, Room G04.614, PO Box 85500, 3584 CX Utrecht, The Netherlands.

E-mail address: A.C.Fluit@umcutrecht.nl (A.C. Fluit).
}

at least $25 \%$ of all milking cows in The Netherlands suffer from clinical mastitis which is not only due to $S$. aureus (32\%) as a causative agent (Lam, 2005). Many different typing methods have been used. From a "gold standard" Pulsed-Field Gel Electrophoresis (PFGE) to Multi Locus Sequence Typing (MLST) and S. aureus-specific staphylococcal Protein A typing known as spa-typing (Enright et al., 2000; Harmsen et al., 2003; Struelens et al., 1992). However, not all methods can be used in all centers because some methods are still limited to well-equipped laboratories. Furthermore, both MLST and spa-typing have insufficient discriminatory power to allow accurate delineation of outbreaks and PFGE is a fingerprinting 
method which makes interlaboratory comparison difficult (van Belkum et al., 1995).

A limited number of molecular studies have been published that explored the population structure and genetic relationships of $S$. aureus causing bovine mastitis (de Sousa et al., 2007; Hata et al., 2006; Jørgensen et al., 2005; Katsuda et al., 2005; Reinoso et al., 2008). The results of our recent study demonstrated that a Multiple Locus Variable Number Tandem Repeats (VNTR) Analysis (MLVA) could be used as a fast, inexpensive, highly discriminatory, reproducible, stable and portable typing method for epidemiological tracing of human S. aureus (Ikawaty et al., 2008). Therefore, we aimed to expand the use of this novel MLVA scheme to $S$. aureus isolated from clinical cases of bovine mastitis and to get insight in their genetic relationship with the human $S$. aureus population.

\section{Methods}

\subsection{Strain collection}

Eighty five S. aureus isolates of clinical or subclinical cases of mastitis were included. Thirty five isolates were obtained by the Faculty of Veterinary Medicine, Utrecht University, Utrecht, The Netherlands from at least 26 farms near Utrecht. Isolates from farms sampled twice were taken at least one year apart. Another 50 isolates were collected by the Animal Health Service in Deventer, The Netherlands for the Central Veterinary Institute, Lelystad, The Netherlands from farms throughout The Netherlands. The sources were individual teat milk samples from dairy cattle from all over the country from clinical or subclinical cases of mastitis. Each isolate from CVI represents one farm, the location of the farms is unknown but they are distributed all over the Netherlands. All isolates were methicillin-susceptible $S$. aureus (MSSA) and collected between 1988 and 2005 .

\subsection{Genomic DNA preparation}

The isolates were grown on blood agar (Trypticase soy agar II containing $5 \%$ sheep blood) overnight at $37^{\circ} \mathrm{C}$ prior to DNA isolation. Preparation of bacterial genomic DNA was performed using the NucleoSpin kit (Macherey-Nagel) following the protocols from the manufacturer with the exception that bacterial pellet is resuspended in buffer T1. In our method, $\mathrm{T} 1$ buffer was replaced by freshly made lysis buffer that contained $20 \mathrm{mM}$ Tris/HCl, 2 mM EDTA, 1\% Triton X-100, and supplemented with lysostaphin, achromopeptidase and RNase.

\subsection{MLVA typing}

A combination of 6 loci (SIRU01, 05, 07, 13, 15 and 21 (spa)) from a previous study by Hardy et al. (2006) were used for MLVA typing. Amplification of SIRUs (Staphylococcal Interspersed Repeat Units) was performed as described before (Ikawaty et al., 2008).

\subsection{Assignment of MLVA type (MT)}

The number of repeats for each locus was determined by subtracting the size of the flanking regions from the size of the amplicon followed by division by size of the repeat (Table 1). The repeat number obtained was rounded up or down to the closest integer copy number. A number string resulted from combination of repeat units from SIRU01, 05, $07,13,15$ and 21 was obtained after calculating the number of repeat units of all loci. This was considered an allelic profile and used for the assignment of an MLVA type (MT).

\subsection{Spa-typing}

Amplification and sequencing of the repeat region of the $S$. aureus Protein A gene ( $s p a$ ) was performed by using a specific primer set as described (Harmsen et al., 2003). The amplicon was sequenced using BigDye terminator version 3.1 on ABI 3100 sequencer (Applied Biosystem). BioNumerics (version 3.5; Applied Maths) was used to analyze the obtained sequences and to assign the spa-types. Novel spa-types were submitted to the Ridom SpaServer database (www.SpaServer.ridom.de).

\subsection{Pulsed field gel electrophoresis}

Bacterial isolates were genotyped by PFGE as described previously (Tenover et al., 1995). Digestion of chromosomal DNA was performed overnight using the restriction enzyme SmaI at a temperature $25^{\circ} \mathrm{C}$. Fragments were separated on $1 \%$ gel. Isolate relatedness was determined using the Tenover criteria (Tenover et al., 1995).

\subsection{MLST analysis}

Determination of the sequence type of $85 \mathrm{~S}$. aureus from bovine mastitis were performed as described (Enright et al., 2000) and data were analyzed using BioNumerics software and the MLST database (www.mlst.net). The outcome of MLST of bovine mastitis $S$. aureus was

Table 1

Size of the MLVA loci, formula for calculating the number of repeat units (RU) per locus, typeability of MLVA and variation in repeat units observed.

\begin{tabular}{llc}
\hline Locus (size in bp) & Formula & Number (\%) PCR negative \\
\hline SIRU01 (55) & $(n-157-30) / 55$ & $0(0.0)$ \\
SIRU05 (60) & $(n-76-78) / 60$ & $85(100)$ \\
SIRU07 (56) & $(n-27-160) / 56$ & $78(91.8)$ \\
SIRU13 (64) & $(n-76-78) / 64$ & $49(47.6)$ \\
SIRU15 (131) & $(n-48-174) / 131$ & $0(0.0)$ \\
SIRU21 (24) & $(n-12-81)-16) / 24$ & $0(0.0)$ \\
\hline
\end{tabular}

$n$ : Size of fragment in bp.

${ }^{a}$ Values are in repeat unit (RU). 
compared with the total population of human S. aureus isolates from our database (data not shown) and the MLST.net database.

\subsection{Comparison of MLVA, spa-typing, MLST and PFGE}

BioNumerics software was used as a tool for clustering the observed MLVA types (MTs) and MLST types (STs). The discriminatory power of the typing methods was calculated by using EpiCompare version 1.0 (Ridom $\mathrm{GmbH}$, Wurzburg, Germany) as well as for the determination of Adjusted Rand index and Wallace's coefficients.

\subsection{Analysis of SIRU05 locus}

Further analysis of the SIRU05 locus was performed by PCR and sequencing using specific primer sets (lysR-For: 5'-GGA AGC AGA TTT AGG TTA TG-3' and fosB-lys-Rev: 5'CCA GTC AAT AGC AAT TTT CC-3' for amplification fragment A; lysR-For: 5'-TTT GTT CAT CTT GGC TTA GG3' and ISRX-lys-Rev: 5'-GGA AGT TAC AAT CAT TTG CG-3' for fragment B) based on reference strain of bovine $S$. aureus RF122 (Fig. 1). DNA sequencing was performed as described for spa-typing.

\section{Results}

\subsection{MLVA}

Among 6 SIRUs used in this typing method, no amplifications were detected for SIRU13, 07 and 05 in $47.6 \%, 91.8 \%$ and $100 \%$ of the cases, respectively (Table 1 ). Variation in the number of repeat units ranged from 0 to 12 repeat units (Table 1 ). Absence of PCR amplification was considered as giving a null allele and is assigned 999 for the repeat number (Table 2). The MLVA typing of 85 isolates produced 18 different allelic profiles or MLVA types. MT102 was most common with 35 isolates, followed by $\operatorname{MT112}(n=17)$, and MT118 $(n=8)$.

\subsection{Spa-typing}

Ten spa-types were obtained from 85 isolates, including 2 new spa-types (t2112 and t2248). Seventy three isolates belonged to three dominant spa-types, t529 $(n=39), \mathrm{t} 543$ $(n=22)$ and $\mathrm{t} 524(n=17)$ (Table 2$)$. The number of repeats obtained by spa-typing for all isolates tested were corresponding with the number of repeats determined by MLVA for SIRU21.

\subsection{Pulsed-Field Gel Electrophoresis}

DNA of 85 isolates was digested with SmaI and showed 26 PFGE profiles, 5 groups of closely related PFGE profiles (5 dominant profiles with 15 related to dominant profiles) and 6 unique PFGE profiles (Fig. 2).

\subsection{MLST}

Sequencing of the seven housekeeping genes of all isolates identified 4 dominant MLST types (STs), ST 504 $(n=24)$, ST $479(n=21)$, ST71 $(n=11)$, ST $151(n=11)$, and 11 STs that have not been described previously in the database at http://saureus.mlst.net (Table 2). A minimum spanning tree of the ST of the 85 isolates compared to the whole population of $S$. aureus showed 3 distinct clusters with bovine mastitis $S$. aureus isolates although some isolates clustered with known human sequence types (Fig. 3).

\subsection{Comparison of MLVA, spa-typing, PFGE and MLST}

Discriminatory power of the four typing methods, PFGE, MLST, spa-typing and MLVA was determined by calculating Simpson's index of diversity with 95\% confidence interval $(\mathrm{CI})$ of the isolates typed by these methods. PFGE showed higher discriminatory power compared to MLST, spa-typing, and MLVA (0.887, $0.831,0.69$ and 0.781 , respectively) (Table 3 ), but the $95 \% \mathrm{CI}$ of MLVA overlapped
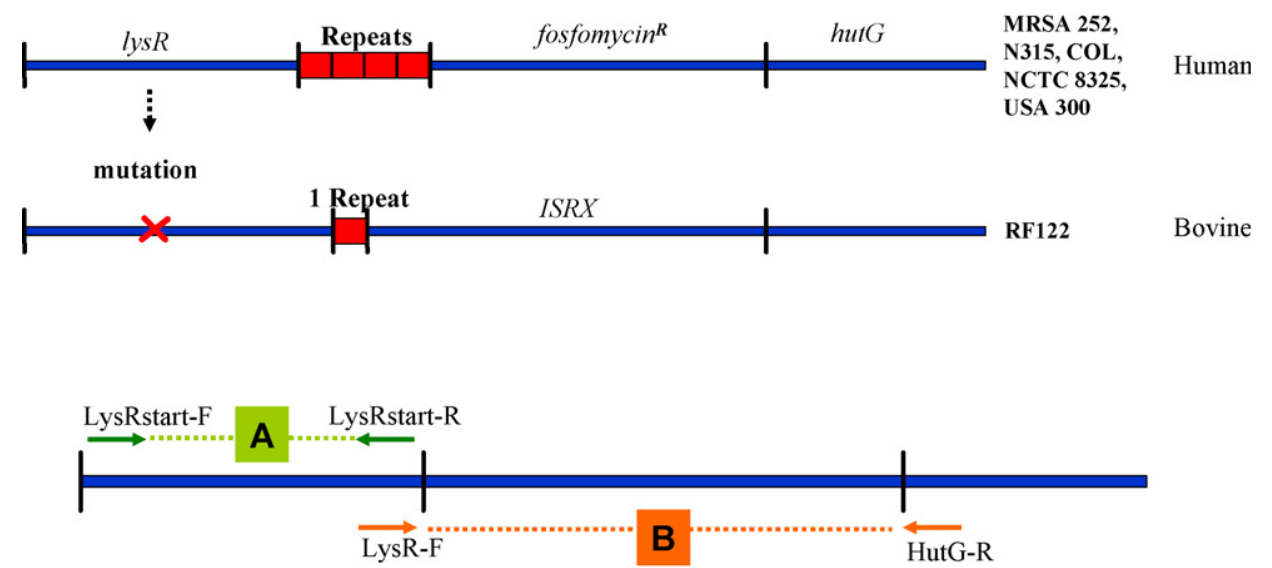

Fig. 1. Strategy for analyzing the region bordering the SIRU05 locus. The bovine S. aureus (BSA) RF122 reference strain showed a different genetic structure compared to human $S$. aureus reference strains. The genetic element at the right of the repeat region consists of a fosfomycin resistance gene in human $S$. aureus and was replaced by insertion site region X (ISRX) in RF122. A single nucleotide mutation in lysR gene region was present in RF122. The primers were designed to detect the presence/absence of a single nucleotide mutation (fragment A) by DNA sequencing and the presence of fosfomycin resistance gene or ISRX structure (fragment B). 
Table 2

Comparison of PFGE, MLST, spa-typing and MLVA results.

\begin{tabular}{|c|c|c|c|c|c|c|c|c|c|c|c|c|c|}
\hline \multirow[t]{2}{*}{ No. } & \multirow[t]{2}{*}{ Isolate } & \multirow[t]{2}{*}{ Date of isolation } & \multirow[t]{2}{*}{ Source } & \multirow[t]{2}{*}{ PFGE profile } & \multirow[t]{2}{*}{ MLST type } & \multirow[t]{2}{*}{ Spa-type } & MLVA type & SIR & & & & & \\
\hline & & & & & & & & 01 & 05 & 07 & 13 & 15 & 21 \\
\hline 1 & S0416 & $04 / 2004$ & FVM & $\mathrm{E}$ & 504 & t529 & 102 & 1 & 999 & 999 & 999 & 0 & 2 \\
\hline 2 & S0409 & $05 / 2005$ & FVM & $\mathrm{E}$ & 151 & t529 & 102 & 1 & 999 & 999 & 999 & 0 & 2 \\
\hline 3 & S0333 & $30 / 09 / 2005$ & CVI & $\mathrm{D}$ & 504 & t529 & 102 & 1 & 999 & 999 & 999 & 0 & 2 \\
\hline 4 & S0334 & $17 / 10 / 2005$ & CVI & $\mathrm{D}$ & 504 & t529 & 102 & 1 & 999 & 999 & 999 & 0 & 2 \\
\hline 5 & S0335 & $17 / 10 / 2005$ & CVI & $\mathrm{E}$ & 151 & t529 & 102 & 1 & 999 & 999 & 999 & 0 & 2 \\
\hline 6 & S0338 & $03 / 10 / 2005$ & CVI & $\mathrm{E}$ & 151 & t529 & 102 & 1 & 999 & 999 & 999 & 0 & 2 \\
\hline 7 & S0341 & $28 / 09 / 2005$ & CVI & D1 & 504 & t529 & 102 & 1 & 999 & 999 & 999 & 0 & 2 \\
\hline 8 & S0343 & $28 / 09 / 2005$ & CVI & $\mathrm{E}$ & 151 & t529 & 102 & 1 & 999 & 999 & 999 & 0 & 2 \\
\hline 9 & S0347 & $10 / 10 / 2005$ & CVI & $\mathrm{D}$ & 504 & t529 & 102 & 1 & 999 & 999 & 999 & 0 & 2 \\
\hline 10 & S0350 & $03 / 10 / 2005$ & CVI & D2 & 151 & t529 & 102 & 1 & 999 & 999 & 999 & 0 & 2 \\
\hline 11 & S0351 & $17 / 10 / 2005$ & CVI & D1 & 151 & t529 & 102 & 1 & 999 & 999 & 999 & 0 & 2 \\
\hline 12 & S0352 & $14 / 10 / 2005$ & CVI & D2 & 151 & t529 & 102 & 1 & 999 & 999 & 999 & 0 & 2 \\
\hline 13 & S0353 & $13 / 10 / 2005$ & CVI & D1 & 151 & t529 & 102 & 1 & 999 & 999 & 999 & 0 & 2 \\
\hline 14 & S0357 & $03 / 10 / 2005$ & CVI & $\mathrm{E}$ & 504 & t529 & 102 & 1 & 999 & 999 & 999 & 0 & 2 \\
\hline 15 & S0358 & $28 / 09 / 2005$ & CVI & $\mathrm{E}$ & 151 & t529 & 102 & 1 & 999 & 999 & 999 & 0 & 2 \\
\hline 16 & S0364 & $30 / 09 / 2005$ & CVI & E & 504 & t529 & 102 & 1 & 999 & 999 & 999 & 0 & 2 \\
\hline 17 & S0365 & $30 / 09 / 2005$ & CVI & D1 & 504 & t529 & 102 & 1 & 999 & 999 & 999 & 0 & 2 \\
\hline 18 & S0367 & $03 / 10 / 2005$ & CVI & D2 & $1122^{b}$ & t529 & 102 & 1 & 999 & 999 & 999 & 0 & 2 \\
\hline 19 & S0368 & $03 / 10 / 2005$ & CVI & $\mathrm{E}$ & 504 & t529 & 102 & 1 & 999 & 999 & 999 & 0 & 2 \\
\hline 20 & S0370 & $28 / 09 / 2005$ & CVI & D1 & $1123^{b}$ & t529 & 102 & 1 & 999 & 999 & 999 & 0 & 2 \\
\hline 21 & S0371 & $17 / 10 / 2005$ & CVI & D & 504 & t529 & 102 & 1 & 999 & 999 & 999 & 0 & 2 \\
\hline 22 & S0374 & $17 / 10 / 2005$ & CVI & D2 & 151 & t529 & 102 & 1 & 999 & 999 & 999 & 0 & 2 \\
\hline 23 & S0375 & $17 / 10 / 2005$ & CVI & D3 & 504 & t529 & 102 & 1 & 999 & 999 & 999 & 0 & 2 \\
\hline 24 & S0376 & $25 / 10 / 2005$ & CVI & $\mathrm{E}$ & 504 & t529 & 102 & 1 & 999 & 999 & 999 & 0 & 2 \\
\hline 25 & S0377 & $25 / 10 / 2005$ & CVI & D1 & 504 & t529 & 102 & 1 & 999 & 999 & 999 & 0 & 2 \\
\hline 26 & S0378 & $25 / 10 / 2005$ & CVI & D & 504 & t529 & 102 & 1 & 999 & 999 & 999 & 0 & 2 \\
\hline 27 & S0379 & $25 / 10 / 2005$ & CVI & $\mathrm{D}$ & 504 & t529 & 102 & 1 & 999 & 999 & 999 & 0 & 2 \\
\hline 28 & S0381 & $25 / 10 / 2005$ & CVI & D & $1124^{\mathrm{b}}$ & t529 & 102 & 1 & 999 & 999 & 999 & 0 & 2 \\
\hline 29 & S0389 & 03/1995 & FVM & $\mathrm{D}$ & 504 & t529 & 102 & 1 & 999 & 999 & 999 & 0 & 2 \\
\hline 30 & S0390 & 03/1997 & FVM & D & $1120^{\mathrm{b}}$ & t529 & 102 & 1 & 999 & 999 & 999 & 0 & 2 \\
\hline 31 & S0396 & $03 / 2002$ & FVM & D1 & 504 & t529 & 102 & 1 & 999 & 999 & 999 & 0 & 2 \\
\hline 32 & S0417 & $04 / 2004$ & FVM & D2 & 504 & t529 & 102 & 1 & 999 & 999 & 999 & 0 & 2 \\
\hline 33 & S0424 & $01 / 2006$ & FVM & D & 504 & t529 & 102 & 1 & 999 & 999 & 999 & 0 & 2 \\
\hline 34 & S0425 & $01 / 2006$ & FVM & $\mathrm{E}$ & 504 & t529 & 102 & 1 & 999 & 999 & 999 & 0 & 2 \\
\hline 35 & S0435 & 1988 & FVM & $\mathrm{D}$ & 504 & t529 & 102 & 1 & 999 & 999 & 999 & 0 & 2 \\
\hline 36 & S0340 & 03/10/2005 & CVI & A & 479 & $\mathrm{t} 543$ & 112 & 2 & 999 & 999 & 2 & 0 & 3 \\
\hline 37 & S0342 & $28 / 09 / 2005$ & CVI & A & 479 & $\mathrm{t} 543$ & 112 & 2 & 999 & 999 & 2 & 0 & 3 \\
\hline 38 & S0344 & $28 / 09 / 2005$ & CVI & A & 479 & t543 & 112 & 2 & 999 & 999 & 2 & 0 & 3 \\
\hline 39 & S0345 & $10 / 10 / 2005$ & CVI & A & 479 & t543 & 112 & 2 & 999 & 999 & 2 & 0 & 3 \\
\hline 40 & S0346 & $10 / 10 / 2005$ & CVI & A & 479 & t543 & 112 & 2 & 999 & 999 & 2 & 0 & 3 \\
\hline 41 & S0348 & $10 / 10 / 2005$ & CVI & A & 479 & t543 & 112 & 2 & 999 & 999 & 2 & 0 & 3 \\
\hline 42 & S0349 & $10 / 10 / 2005$ & CVI & A & 479 & t543 & 112 & 2 & 999 & 999 & 2 & 0 & 3 \\
\hline 43 & S0354 & $03 / 10 / 2005$ & CVI & A & 479 & t543 & 112 & 2 & 999 & 999 & 2 & 0 & 3 \\
\hline 44 & S0355 & $03 / 10 / 2005$ & CVI & A & 479 & $\mathrm{t} 543$ & 112 & 2 & 999 & 999 & 2 & 0 & 3 \\
\hline 45 & S0356 & $03 / 10 / 2005$ & CVI & A & 479 & t543 & 112 & 2 & 999 & 999 & 2 & 0 & 3 \\
\hline 46 & S0360 & $28 / 09 / 2005$ & CVI & A & 479 & t543 & 112 & 2 & 999 & 999 & 2 & 0 & 3 \\
\hline 47 & S0361 & $28 / 09 / 2005$ & CVI & A & 479 & $\mathrm{t} 543$ & 112 & 2 & 999 & 999 & 2 & 0 & 3 \\
\hline 48 & S0369 & 03/10/2005 & CVI & A & $1118^{b}$ & $\mathrm{t} 543$ & 112 & 2 & 999 & 999 & 2 & 0 & 3 \\
\hline 49 & S0372 & $17 / 10 / 2005$ & CVI & A & 479 & t543 & 112 & 2 & 999 & 999 & 2 & 0 & 3 \\
\hline 50 & S0373 & $17 / 10 / 2005$ & CVI & A & $1118^{\mathrm{b}}$ & $\mathrm{t} 543$ & 112 & 2 & 999 & 999 & 2 & 0 & 3 \\
\hline 51 & S0414 & $09 / 2003$ & FVM & A & 479 & $\mathrm{t} 543$ & 112 & 2 & 999 & 999 & 2 & 0 & 3 \\
\hline 52 & S0422 & $01 / 2005$ & FVM & A & 479 & $\mathrm{t} 543$ & 112 & 2 & 999 & 999 & 2 & 0 & 3 \\
\hline 53 & S0398 & $10 / 1998$ & FVM & A & 479 & t543 & 114 & 2 & 999 & 999 & 1 & 0 & 3 \\
\hline 54 & S0332 & $12 / 10 / 2005$ & CVI & $\mathrm{A} 1$ & 479 & t543 & 114 & 2 & 999 & 999 & 1 & 0 & 3 \\
\hline 55 & S0339 & $03 / 10 / 2005$ & CVI & A & 479 & t543 & 114 & 2 & 999 & 999 & 1 & 0 & 3 \\
\hline 56 & S0391 & $04 / 2002$ & FVM & A & 479 & t543 & 114 & 2 & 999 & 999 & 1 & 0 & 3 \\
\hline 57 & S0388 & 1994 & FVM & F3 & $1119^{b}$ & $\mathrm{t} 524$ & 118 & 4 & 999 & 999 & 3 & 1 & 2 \\
\hline 58 & S0401 & 01/1999 & FVM & B3 & $1125^{\mathrm{b}}$ & $\mathrm{t} 524$ & 118 & 4 & 999 & 999 & 3 & 1 & 2 \\
\hline 59 & S0427 & 1990 & FVM & B & $1129^{b}$ & $\mathrm{t} 524$ & 118 & 4 & 999 & 999 & 3 & 1 & 2 \\
\hline 60 & S0428 & 1989 & FVM & $\mathrm{F}$ & 71 & $\mathrm{t} 524$ & 118 & 4 & 999 & 999 & 3 & 1 & 2 \\
\hline 61 & S0429 & 1989 & FVM & G & 71 & $\mathrm{t} 524$ & 118 & 4 & 999 & 999 & 3 & 1 & 2 \\
\hline 62 & S0430 & 1989 & FVM & $\mathrm{F} 1$ & 71 & $\mathrm{t} 524$ & 118 & 4 & 999 & 999 & 3 & 1 & 2 \\
\hline 63 & S0432 & 1989 & FVM & B & 71 & $\mathrm{t} 524$ & 118 & 4 & 999 & 999 & 3 & 1 & 2 \\
\hline 64 & S0412 & $06 / 2003$ & FVM & B & 71 & t524 & 118 & 4 & 999 & 999 & 3 & 1 & 2 \\
\hline 65 & S0362 & $30 / 09 / 2005$ & CVI & B6 & 71 & $\mathrm{t} 524$ & 125 & 5 & 999 & 999 & 3 & 1 & 2 \\
\hline 66 & S0433 & 1989 & FVM & B1 & 71 & $\mathrm{t} 524$ & 125 & 5 & 999 & 999 & 3 & 1 & 2 \\
\hline 67 & S0434 & 1989 & FVM & B & 71 & $\mathrm{t} 524$ & 125 & 5 & 999 & 999 & 3 & 1 & 2 \\
\hline 68 & S0411 & $06 / 2003$ & FVM & B2 & $1127^{b}$ & t524 & 125 & 5 & 999 & 999 & 3 & 1 & 2 \\
\hline
\end{tabular}


Table 2 (Continued)

\begin{tabular}{|c|c|c|c|c|c|c|c|c|c|c|c|c|c|}
\hline \multirow[t]{2}{*}{ No. } & \multirow[t]{2}{*}{ Isolate } & \multirow[t]{2}{*}{ Date of isolation } & \multirow[t]{2}{*}{ Source } & \multirow[t]{2}{*}{ PFGE profile } & \multirow[t]{2}{*}{ MLST type } & \multirow[t]{2}{*}{ Spa-type } & \multirow[t]{2}{*}{ MLVA type } & \multicolumn{6}{|c|}{ SIRU $^{\mathrm{a}}$} \\
\hline & & & & & & & & 01 & 05 & 07 & 13 & 15 & 21 \\
\hline 69 & S0413 & $07 / 2003$ & FVM & B5 & $1128^{b}$ & $\mathrm{t} 524$ & 125 & 5 & 999 & 999 & 3 & 1 & 2 \\
\hline 70 & S0420 & $10 / 2004$ & FVM & D1 & 504 & t529 & 125 & 5 & 999 & 999 & 3 & 1 & 2 \\
\hline 71 & S0418 & $08 / 2005$ & FVM & E1 & 504 & t529 & 104 & 1 & 999 & 999 & 3 & 1 & 2 \\
\hline 72 & S0410 & $05 / 2003$ & FVM & B4 & $1126^{\mathrm{b}}$ & $\mathrm{t} 524$ & 104 & 1 & 999 & 999 & 3 & 1 & 2 \\
\hline 73 & S0419 & $11 / 2004$ & FVM & $\mathrm{C}$ & 71 & $\mathrm{t} 524$ & 79 & 6 & 999 & 999 & 3 & 1 & 2 \\
\hline 74 & S0421 & $11 / 2004$ & FVM & D1 & 151 & t529 & 79 & 6 & 999 & 999 & 3 & 1 & 2 \\
\hline 75 & S0363 & $30 / 09 / 2005$ & CVI & F4 & 71 & $\mathrm{t} 524$ & 76 & 5 & 999 & 999 & 3 & 0 & 2 \\
\hline 76 & S0436 & 1990 & FVM & $\mathrm{H}$ & 97 & t2174 & 82 & 6 & 999 & 2 & 5 & 2 & 6 \\
\hline 77 & S0387 & 1994 & FVM & D1 & 504 & t529 & 106 & 1 & 999 & 3 & 999 & 0 & 2 \\
\hline 78 & S0426 & 1990 & FVM & B2 & 97 & $\mathrm{t} 1236$ & 108 & 1 & 999 & 2 & 5 & 3 & 10 \\
\hline 79 & S0366 & $03 / 10 / 2005$ & CVI & I & $1121^{\mathrm{b}}$ & $\mathrm{t} 127$ & 110 & 1 & 999 & 3 & 2 & 2 & 7 \\
\hline 80 & S0392 & $04 / 2002$ & FVM & A & 479 & $\mathrm{t} 2248$ & 116 & 2 & 999 & 999 & 1 & 0 & 6 \\
\hline 81 & S0337 & $13 / 10 / 2005$ & CVI & B2 & 97 & $\mathrm{t} 2112$ & 120 & 4 & 999 & 2 & 5 & 2 & 11 \\
\hline 82 & S0431 & 1989 & FVM & F2 & 97 & t521 & 121 & 4 & 999 & 3 & 3 & 2 & 12 \\
\hline 83 & S0336 & $17 / 10 / 2005$ & CVI & $\mathrm{J}$ & 71 & $\mathrm{t} 524$ & 123 & 4 & 999 & 999 & 2 & 1 & 2 \\
\hline 84 & S0423 & $11 / 2005$ & FVM & A & 479 & t543 & 127 & 5 & 999 & 999 & 3 & 1 & 3 \\
\hline 85 & S0393 & $08 / 2001$ & FVM & K & 124 & t224 & 129 & 5 & 999 & 3 & 5 & 1 & 8 \\
\hline
\end{tabular}

CVI: Central Veterinary Institute, Lelystad, The Netherlands.

FVM: Faculty of Veterinary Medicine, Utrecht University, Utrecht, The Netherlands. 999: no amplification of SIRU.

a Values are in repeat units (RU).

b New MLST type.

with those of spa-typing and MLST. It is remarkable that all 3 major spa-types contained multiple ST (Table 2).

\subsection{Analysis of the neighboring region of SIRU05}

SIRU05 could not be amplified for any of the isolates. Therefore, the presence of sequences bordering SIRU05 was investigated. In human-derived SIRU05-positive strains SIRU05 is flanked by a LysR regulatory protein family gene at one side and a fosfomycin resistance encoding gene $(f o s B)$ followed by the hutG gene which putatively encodes a formimidoylglutamase. In the sequenced bovine strain RF122 the fosfomycin resistance gene is replaced by insertion sequence ISRX. One primer set was used to amplify the lysR region (fragment A) and a second set was used to amplify the region between the lys $R$ gene and hutG (Fig. 1). All isolates were positive for amplification of fragment A and B. Sequencing of fragment $A$ and $B$ of 10 isolates showed a single nucleotide mutation in the lysR gene resulting a premature stop-codon and the absence of ISRX. A new primer designed in the hutG gene which was combined with the original primer of SIRU05 in the lysR gene did not show differences in the number of repeat units for this locus.

\subsection{The congruence between PFGE, MLST, spa-typing and MLVA}

Adjusted Rand's and Wallace's coefficients were calculated to explore the concordance between typing methods (Tables 4 and 5). The Adjusted Rand's coefficient for the comparison of the clustering by MLVA and PFGE, MLVA and MLST, and MLVA and spa-typing was 0.385, 0.442 and 0.758 , respectively. Considering MLVA as the test typing method for comparison, the value of Wallace's coefficients showed that MLVA could only poorly predict the PFGE and MLST type. The probability of two strains having the same MLVA type and sharing the same spa-type was $99 \%$
(Wallace's coefficient 0.991), whereas the reverse was reasonably predictive (Wallace's coefficient 0.699 ). This finding reflects that MLVA was less discriminatory than MLST and PFGE.

We observed variation of spa-type and MT within the same MLST type as shown by ST97 and 479 isolates, although an identical spa-type isolates also showed variation of STs that were closely related (single locus variants or SLVs) to possibly related (3 loci different).

Identical t524 isolates had new STs: ST1119, 1125, 1126,1127 , and 1129 that were SLVs and ST1128 that was a double locus variant (DLV) of ST71.

\section{Discussion}

MLVA has shown great potential for fast and reliable typing of pathogenic bacteria. Our previous study demonstrated that a newly developed MLVA scheme for human $S$. aureus had higher discriminatory power compared to PFGE, MLST and spa-typing (Ikawaty et al., 2008). A major advantage of the proposed MLVA scheme is that it requires only simple laboratory equipment and is fast and relatively cheap to perform. This scheme provides better and more timely access to typing of bovine mastitis. This allows more adequate surveillance of mastitis and the identification of particular virulent or epidemic strains. Early recognition of these strains may help to initiate more timely therapy and other interventions to prevent further spread. In this study, we extended the use of the MLVA typing scheme for human $S$. aureus to bovine $S$. aureus from clinical mastitis. Eighty five isolates represented regional $(n=35)$ and national $(n=50) S$. aureus isolates. The isolates were not considered to belong to local outbreaks as $90 \%$ of the isolates were obtained on different farms whereas the remaining isolates were obtained at least one year apart when sampled on the same farm. The scheme showed good typeability although 3 loci (SIRU05, 07 and 13) were not always amplified. SIRU21 had the most variance in the 

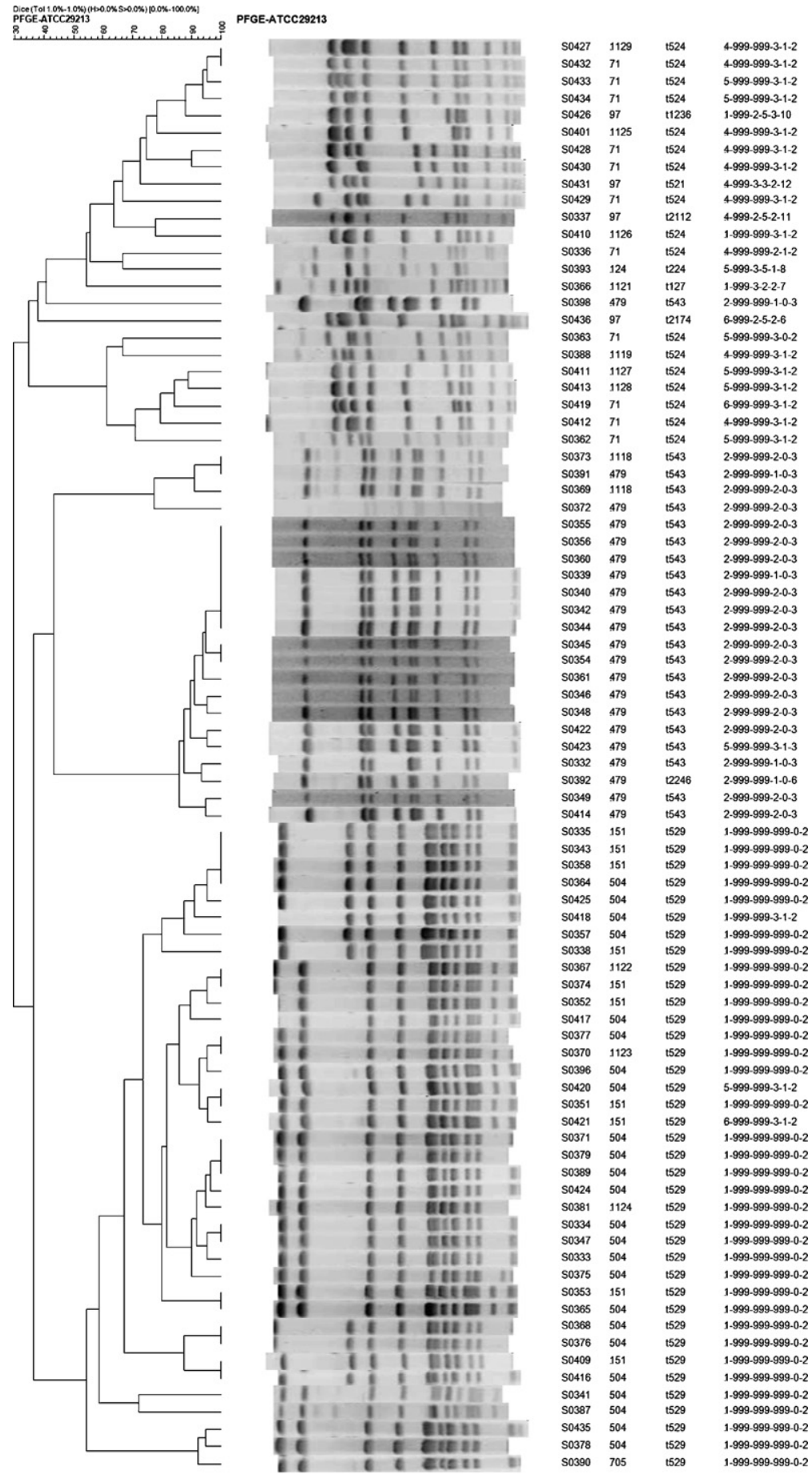

Fig. 2. Dendrogram containing PFGE patterns of 85 MSSA strains collected from bovine. At the $50 \%$ similarity level, seven branches are distinguished. ID: isolate ID; ST: MLST type.

number of repeat units, which is important in typing by MLVA.

We observed no particular difference between regionally and nationally obtained $S$. aureus bovine mastitis strains in terms of PFGE profile, MLST and spa-types, except for one MLVA type (MT 118) that was only present among regionally obtained isolates, but due to the limited number of isolates further analysis using more samples is needed.

The number of repeat units obtained by amplification of SIRU21, having the smallest repeat unit, agreed completely with the number of repeats obtained by DNA sequencing for spa-typing indicating the reliability of the determination of the number of repeat units. 


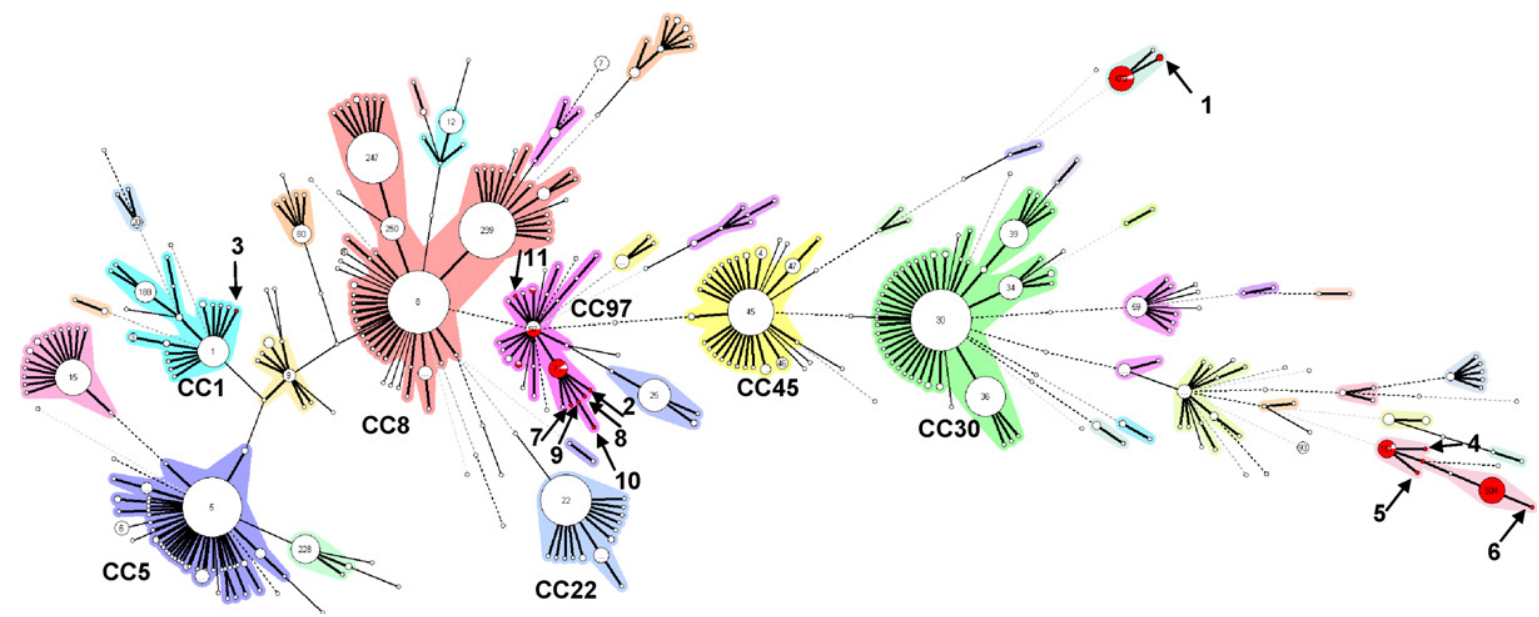

Fig. 3. Minimum spanning tree of S. aureus based on MLST. Five major clonal complexes are present within the S. aureus population: CC5, CC8, CC22, CC30 and CC45. Each circle represents a different MLST type. Single locus variant STs are connected by a thick line, double locus variant STs by a thin line, triple locus variant STs by a dark grey dashed line, and STs with more than 3 loci variant are connected by a light grey dashed line. Red circles indicate $S$. aureus isolated from bovine mastitis. Arrows indicated new MLST types observed in this study: 1: ST 1118; 2: ST 1119; 3: ST 1121; 4: ST 1122; 5: ST 1123; 6: ST 1124; 7: ST 1125; 8: ST 1126; 9: ST 1127; 10: ST 1128; 11: ST 1129.

SIRU05 was analyzed more in depth, since it could not be amplified in any of the bovine isolates including 4 isolates with ST97 that were already known as possibly human derived (Smith et al., 2005a; Sung et al., 2008). PCR of the region neighboring SIRU05 demonstrated that all isolates were fragment $A$ and $B$ positive. Sequencing of the fragments of 10 isolates showed that ISRX and the fosfomycin resistance element were lacking. The difference in the genetic element bordering the repeats of SIRU05 explains the negative result of SIRU05 PCR, since the primer chosen in the fosfomycin resistance element could not anneal to the ISRX element or the hutG gene. Amplification of SIRU05 with a primer chosen in hutG showed no variation in the number of repeat units. SIRU05 does not contribute to the discrimination of the isolates tested and can be omitted from the scheme for bovine mastitis isolates. It should be noted that the primer sets used in this MLVA scheme were developed based on human S. aureus.

LysR is a member of the largest family of bacterial activator/regulator proteins (Henikoff et al., 1988; Zaim and Kierzek, 2003). The LysR protein contains a substrate recognition site and a helix-turn-helix DNA-binding motif crucial for protein-DNA interaction (Zaim and Kierzek, 2003). In bovine $S$. aureus a stop-codon is present, which prevents transcription of the substrate domain. This should inactivate LysR leading to different expression patterns in bovine strains compared to human strains.
A minimum spanning tree based on MLST showed that clustering of bovine S. aureus was different than for isolates from the human population. The difference between human and bovine derived isolates has been suggested to be caused by tissue specificity by some authors (Gilbert et al., 2006; Smith et al., 2005a) while most authors assumed host specificity among $S$. aureus clones (de Sousa et al., 2007; Kapur et al., 1995). Two of the three dominant MLST types (ST151 and 504) were clustered together while ST479 was distantly related to ST151 and ST504. They were not related to human-derived S. aureus. Eleven isolates from both regional and national origin were ST71 which is known to be a bovine-associated strain from The Netherlands (Smith et al., 2005a). Interestingly, the 11 new STs isolates fell into a cluster which was not related to the known bovine STs. Little sharing of strains between the bovine and human population has been reported (Kapur et al., 1995). A similar finding was made by Rabello et al. for Brazilian isolates (Rabello et al., 2007).

The ST or clonal complexes (CC) of bovine mastitis isolates from The Netherlands differed from those described elsewhere with the exception of 4 isolates (S0337, S0426, S0431 and S0436) that belonged to CC97. CC97 isolates have previously been reported to be obtained from humans (Feil et al., 2003). In Brazil CC97 and CC127 were predominant (Rabello et al., 2007). CC97 was also present in isolates from the UK, the USA and Chile (Smith et al., 2005a,b). A different set of STs was obtained from

Table 3

Simpson's index of diversity and 95\% confidence interval.

\begin{tabular}{llll}
\hline Typing method & Number of different types & Discriminatory index & 95\% confidence interval \\
\hline PFGE & 26 & 0.887 & $0.847-0.926$ \\
MLST & 18 & 0.831 & $0.787-0.875$ \\
Spa-typing & 10 & 0.69 & $0.627-0.752$ \\
MLVA & 18 & 0.781 & $0.71-0.852$ \\
\hline
\end{tabular}

Total of 85 isolates tested. 
Table 4

Adjusted Rand's coefficients for the methods used to characterize the 85 bovine mastitis Staphylococcus aureus isolates.

\begin{tabular}{lllll}
\hline Typing method & PFGE & MLST & Spa-typing & MLVA \\
\hline PFGE & - & & & \\
MLST & 0.479 & - & & \\
Spa-typing & 0.405 & 0.583 & - & - \\
MLVA & 0.385 & 0.442 & 0.758 & \\
\hline
\end{tabular}

Table 5

Wallace's coefficients for the methods used to characterize 85 bovine mastitis Staphylococcus aureus isolates.

\begin{tabular}{lllll}
\hline Typing method & PFGE & MLST & Spa-typing & MLVA \\
\hline PFGE & - & 0.684 & 0.941 & 0.699 \\
MLST & 0.459 & - & 0.957 & 0.63 \\
Spa-typing & 0.344 & 0.521 & - & 0.699 \\
MLVA & 0.362 & 0.486 & 0.991 & - \\
\hline
\end{tabular}

milk in Norway where ST130 (CC3), ST133 and ST132 (CC1) were predominant (Jørgensen et al., 2005).

The highest discriminatory power was obtained by PFGE followed by MLST, MLVA and spa-typing. Because SIRU05 yielded no usable data a lower discriminatory power of MLVA compared to the spa-typing was expected. But the results demonstrated that MLVA had a higher Simpson's index of diversity than spa-typing.

We observed variation in related STs within the same spa-type as shown in Table 2. The limited number of spatypes might suggest selective pressure on the spa gene and it may explain their maintenance in the population of bovinederived S. aureus. This finding suggests that spa-typing is not a useful method to compare bovine mastitis isolates.

Transfer of antibiotic resistance from human to animal isolates or the other way around has been a major concern (Pesavento et al., 2007; Zadoks et al., 2002). Host specificity of clones may reduce the chance that human-derived antibiotic-resistant $S$. aureus isolates are transmitted to cattle, although bovine mastitis may occasionally be caused by human-derived isolates. Typing of $S$. aureus from bovine mastitis will be required to monitor potentially changing population dynamics. Based on our data PFGE, MLST, and MLVA are adequate typing methods for international studies and local studies of bovine $S$. aureus isolates involved in mastitis. However, PFGE and MLST are more time-consuming and/or expensive than MLVA.

\section{References}

de Sousa, A.M., Parente, C.E., Vieira-da-Motta, O., Bonna, I.C., Silva, D.A., de Lencastre, H., 2007. Characterization of Staphylococcus aureus isolates from buffalo, bovine, ovine, and caprine milk samples collected in Rio de Janeiro State, Brazil. Appl. Environ. Microbiol. 73, 3845-3849.

Enright, M.C., Day, N.P., Davies, C.E., Peacock, S.J., Spratt, B.G., 2000. Multilocus sequence typing for characterization of methicillin-resistant and methicillin-susceptible clones of Staphylococcus aureus. J. Clin. Microbiol. 38, 1008-1015.

Feil, E.J., Cooper, J.E., Grundmann, H., Robinson, D.A., Enright, M.C., Peacock, S.J., Smith, J.M., Murphy, M., Spratt, B.G., Moore, C.E., Day, N.P., 2003. How clonal is Staphylococcus aureus? J. Bacteriol. 185, 3307-3316.

Gilbert, F.B., Fromageau, A., Gelineau, L., Poutrel, B., 2006. Differentiation of bovine Staphylococcus aureus isolates by use of polymorphic tandem repeat typing. Vet. Microbiol. 117, 297-303.
Hardy, K.J., Oppenheim, B.A., Gossain, S., Gao, F., Hawkey, P.M., 2006. Use of variations in staphylococcal interspersed repeat units for molecular typing of methicillin-resistant Staphylococcus aureus strains. J. Clin. Microbiol. 44, 271-273.

Harmsen, D., Claus, H., Witte, W., Rothganger, J., Claus, H., Turnwald, D., Vogel, U., 2003. Typing of methicillin-resistant Staphylococcus aureus in a university hospital setting by using novel software for spa repeat determination and database management. J. Clin. Microbiol. 41, 5442-5448.

Hata, E., Katsuda, K., Kobayashi, H., Ogawa, T., Endô, T., Eguchi, M., 2006. Characteristics and epidemiologic genotyping of Staphylococcus aureus isolates from bovine mastitic milk in Hokkaido, Japan. J. Vet. Med. Sci. $68,165-170$.

Henikoff, S., Haughn, G.W., Calvo, J.M., Wallace, J.C., 1988. A large family of bacterial activator proteins. Proc. Natl. Acad. Sci. U.S.A. 85, 66026606.

Ikawaty, R., Willems, R.J.L., Box, A.T.A., Verhoef, J., Fluit, A.C., 2008. A novel multiple locus variable number tandem repeat (VNTR) analysis for rapid molecular typing of human Staphylococcus aureus. J. Clin. Microbiol. 46, 3147-3151.

Jørgensen, H.J., Mørk, T., Caugant, D.A., Kearns, A., Røvik, L.M., 2005. Genetic variation among Staphylococcus aureus strains from Norwegian bulk milk. Appl. Environ. Microbiol. 71, 8352-8361.

Kapur, V., Sischo, W.M., Greer, R.S., Whittam, T.S., Musser, J.M., 1995. Molecular population genetic analysis of Staphylococcus aureus recovered from cows. J. Clin. Microbiol. 33, 376-380.

Katsuda, K., Hata, E., Kobayashi, H., Kohmoto, M., Kawashima, K., Tsunemitsu, H., Eguchi, M., 2005. Molecular typing of Staphylococcus aureus isolated from bovine mastitic milk on the basis of toxin genes and coagulase gene polymorphisms. Vet. Microbiol. 105, 301-305.

Lam, T., 2005. Five year project to reduce mastitis in The Netherlands. Mastitis Newslett. 26, 33.

Pesavento, G., Ducci, B., Comodo, N., Lo Nostro, A., 2007. Antimicrobial resistance profile of Staphylococcus aureus isolated from raw meat: a research for methicillin resistant Staphylococcus aureus (MRSA). Food Control 18, 196-200.

Rabello, R.F., Moreira, B.M., Lopes, R.M.M., Teixeira, L.M., Riley, L.W., Castro, A.C.D., 2007. Multilocus sequence typing of Staphylococcus aureus isolates recovered from cows with mastitis in Brazilian dairy herds. J. Med. Microbiol. 56, 1505-1511.

Reinoso, A.B., El-Sayed, A., Lämmler, C., Bogni, C., Zschöck, M., 2008. Genotyping of Staphylococcus aureus isolated from humans, bovine subclinical mastitis and food samples in Argentina. Microbiol. Res. $163,314-322$

Smith, E.M., Green, L.E., Medley, G.F., Bird, H.E., Fox, L.K., Schukken, Y.H. Kruze, J.V., Bradley, A.J., Zadoks, R.N., Dowson, C.G., 2005a. Multilocus sequence typing of intercontinental bovine Staphylococcus aureus isolates. J. Clin. Microbiol. 43, 4737-4743.

Smith, E.M., Green, L.E., Medley, G.F., Bird, H.E., Dowson, C.G., 2005b. Multilocus sequence typing of Staphylococcus aureus isolated from high-somatic-cell-count cows and the environment of an organic dairy farm in the United Kingdom. J. Clin. Microbiol. 43, 4731-4736.

Struelens, M.J., Deplano, A., Godard, C., Maes, N., Serruys, E., 1992 Epidemiologic typing and delineation of genetic relatedness of methicillin resistant Staphylococcus aureus by macrorestriction analysis of genomic DNA by using pulsed-field gel electrophoresis. J. Clin. Microbiol. 30, 2599-2605.

Sung, J.M.-L., Lloyd, D.H., Lindsay, J.A., 2008. Staphylococcus aureus host specificity: comparative genomics of human versus animal isolates by multi-strain microarray. Microbiology 154, 1949-1959.

Tenover, F.C., Arbeit, R.D., Goering, R.V., Mickelsen, P.A., Murray, B.E., Persing, D.H., Swaminathan, B., 1995. Interpreting chromosomal DNA restriction patterns produced by pulsed-field gel electrophoresis: criteria for bacterial strain typing. J. Clin. Microbiol. 33, 2233-2239.

van Belkum, A., Kluytmans, J.A., van Leeuwen, W., Bax, R., Quint, W. Peters, E., Fluit, A.C., Vandenbroucke-Grauls, C.M., van den Brule, A. Koeleman, H., Melchers, W., Meis, J., Elaichouni, A., Vaneechoutte, M. Moonens, F., Maes, N., Struelens, M.J., Tenover, F.C., Verbrugh, H. 1995. Multicenter evaluation of arbitrarily primed PCR for typing of Staphylococcus aureus strains. J. Clin. Microbiol. 33, 1537-1547.

Zadoks, R.N., van Leeuwen, W.B., Kreft, D., Fox, L.K., Barkema, H.W., Schukken, Y.H., van Belkum, A., 2002. Comparison of Staphylococcus aureus isolates from bovine and human skin, milking equipment, and bovine milk by phage typing, pulsed-field gel electrophoresis, and binary typing. J. Clin. Microbiol. 40, 3894-3902.

Zaim, J., Kierzek, A.M., 2003. The structure of full-length LysR-type transcriptional regulators. Modeling of the full-length OxyR transcriptional factor dimer. Nucleic Acids Res. 31, doi:10.1093/nar/ gkg234. 